\title{
In situ dynamic transmission electron microscopy characterization of liquid- mediated crystallization of amorphous Ge
}

\author{
G. Egan, ${ }^{1}$ T.T. Rahn, ${ }^{2}$ A.J. Rise, ${ }^{2}$ H.-Y. Cheng, ${ }^{3}$ S. Raoux, ${ }^{4}$ G.H. Campbell, ${ }^{1}$ and M.K. Santala, ${ }^{2, a)}$ \\ ${ }^{1}$ Materials Science Division, Lawrence Livermore National Laboratory, Livermore, CA, 94551, USA \\ ${ }^{2}$ Department of Mechanical, Industrial, and Manufacturing Engineering, Oregon State University, \\ Corvallis, OR, 97331, USA \\ ${ }^{3}$ IBM/Macronix PCRAM Joint Project, Macronix Emerging Central Laboratory, Macronix International \\ Co., Hsinchu, Taiwan \\ ${ }^{4}$ Helmholtz-Zentrum Berlin für Materialien und Energie GmbH, D-14109, Berlin, Germany
}

\section{ABSTRACT}

Crystal growth rates during laser-induced, liquid-mediated crystallization of amorphous Ge were measured with movie-mode dynamic transmission electron microscopy (MM-DTEM), a photo-emission microscopy technique with nanosecond-scale time resolution. Films of 50-nm thick amorphous Ge were crystallized using a 12-ns laser pulse with a Gaussian spatial profile, which established high local temperature gradients in the specimen. Crystallization proceeded by formation of a central zone with a high nucleation rate (Zone I), followed by liquid-mediated outward growth of columnar grains (Zone II), followed by spiraling growth (Zone III) until the crystallization halted in cooler parts of the specimen. Zone II growth was imaged for several laser pulse energies with 20-ns electron pulses with 95 ns between frames. A thin liquid layer between the solid amorphous phase and the advancing crystallization front during Zone II growth was imaged. The Zone II growth rate for each experiment remained nearly constant although the crystallization front passes through a large temperature gradient. Measured growth rates ranged from 5.7 to $13.6 \mathrm{~m} / \mathrm{s}$, consistent with transient-liquid-layer mediated growth rather than solid-state growth. In contrast with a previous report, the growth rate did not increase systematically with laser energy or absorbed energy. The new results, together with previously reported data, suggest both sets of experiments were conducted under conditions where the growth rate saturates near its maximum value. A phenomenological model based on the concept of an upper and lower threshold temperatures for the Zone II growth was fitted to the data from these experiments and previous MM-DTEM crystallization experiments.

a) Author to whom correspondence should be addressed. Electronic mail: melissa.santala@oregonstate.edu. 


\section{INTRODUCTION}

Laser annealing is used to process amorphous semiconductor thin films for a variety of applications including thin-film solar cells ${ }^{1,2}$ and metal-oxide-semiconductor field-effect transistors. ${ }^{3}$ Under the correct heat loss conditions, laser annealing induces crystal growth mediated by a thin, transient liquid layer that enables far more rapid growth than could occur in purely solid-state crystallization of the amorphous solid. Growth rates on the order of $10 \mathrm{~m} / \mathrm{s}$

have been measured by multiple groups for laser-induced crystallization of Ge thin films. ${ }^{4-8}$ This growth mechanism results in different microstructure than low temperature isothermal crystallization and can yield thin semiconductor films with large grain sizes ${ }^{9}$ and relatively low sheet resistance. Here, the crystal growth rate in amorphous Ge thin films is measured where growth is extremely rapid and mediated by a transient liquid layer between the solid amorphous and crystalline phases using movie-mode dynamic transmission electron microscopy (MMDTEM). The high spatio-temporal resolution of this technique enables the progression of the liquid-mediated growth to be observed and for growth rates to be measured in regions where high temperature gradients $\left(>10^{10} \mathrm{~K} / \mathrm{m}\right)$ are established by the Gaussian laser pulse.

\section{A. Liquid-mediated crystal growth in amorphous semiconductors}

The mechanism for liquid-mediated growth of amorphous Ge was proposed in the 1970's by Bagley and Chen ${ }^{10}$ and Spaepen and Turnbull. ${ }^{11}$ They hypothesized that amorphous semiconductors may undergo a first order solid-to-liquid phase transition at a temperature well under the equilibrium melting temperature of the crystalline phase, $\mathrm{T}_{\mathrm{m}}$. Bagley and Chen used calorimetric data to estimate that the amorphous-solid to liquid transition temperature, $T_{\mathrm{ma}}$, for amorphous Ge to be $\sim 969 \mathrm{~K} .{ }^{10}$ The presence of a liquid with a depressed melting temperature was invoked to explain extremely high nucleation rates and crystal growth rates ${ }^{10}$ observed 
during laser annealing, which exceeds - by orders of magnitude - what is plausible with purely solid-state diffusive growth.

Rapid crystallization of amorphous semiconductor film mediated by a very highlyundercooled, transient liquid layer has been called "explosive" crystallization ${ }^{12}$ because it may generate visible light and considerable heat. ${ }^{13,14}$ Explosive crystallization has been studied in $\mathrm{Si},{ }^{15-18} \mathrm{Ge},{ }^{4-9,12,14,16,19-26}$ and $\mathrm{Sb}^{13,27-29}$ There is also evidence that Sb-rich alloys such as GeSb 6 Te may undergo rapid liquid-mediated growth. ${ }^{30}$ During liquid-mediated crystallization, a thin liquid layer ${ }^{15,18}$ forms ahead of the crystal growth front by the heat of transformation released during the crystallization. The enthalpy of crystallization $\left(5.10 \times 10^{6} \mathrm{~J} / \mathrm{kg}\right)^{31}$ released raises the adjacent amorphous material above the $\mathrm{T}_{\mathrm{ma}}$, but the newly formed liquid quickly crystallizes because it is highly undercooled ( $\mathrm{T}_{\mathrm{ma}}$ for Ge is $\sim 242 \mathrm{~K}$ below $\mathrm{T}_{\mathrm{m}}$ ). The crystallization kinetics and the resulting microstructures are affected by the local temperature, temperature gradients, and heat flow, which are determined by the heating method and specimen geometry. If the substrate temperature is high enough and heat loss to the surroundings is limited, then the liquid layer may be continually re-generated and liquid-mediated crystallization may be maintained across an entire specimen. ${ }^{4-6}$ Experimental studies of the role of heat loss in the laser-induced, liquid-mediated crystal growth rate performed by Chojnacka and Thompson ${ }^{4,5}$ and Grigoropoulus et al. ${ }^{6}$ showed a broad range of temperatures where the growth rate saturates at a maximum value. Both groups initiated crystallization in thin Ge films on substrates pre-heated to temperatures ranging from $570 \mathrm{~K}$ to $850 \mathrm{~K}$. They found that, above a threshold temperature, the growth rate has little dependence on the substrate temperature, though the maximal growth rate observed by Chojnacka was between 12 and $13 \mathrm{~m} / \mathrm{s}^{5}$ whereas it was between 8 and $9 \mathrm{~m} / \mathrm{s}$ in Grigoropoulos' work. ${ }^{6}$ For films in the range of $1.3-3.0 \mu \mathrm{m}$ thick, Chojnacka ${ }^{5}$ found that film 
thickness does not impact the maximum liquid-mediated crystal growth rate, but does impact the threshold temperature which was $680 \mathrm{~K}$ for the $3.0 \mu \mathrm{m}$ films and increased to $740 \mathrm{~K}$ for $1.3 \mu \mathrm{m}$ thick films. Molecular dynamics simulations for liquid-mediated crystallization of both Si and $\mathrm{Ge}^{15}$ similarly showed that under heat loss conditions where steady state growth is maintained, the growth rate tends toward a maximal value for a range of crystal-liquid and liquid-amorphous interface temperatures. The thickness of the liquid layer was found to vary, compensating for variations in heat loss conditions, resulting in a self-regulating process.

\section{B. In situ transmission electron microscopy of liquid-mediated crystal growth of amorphous Ge}

Transmission electron microscopy (TEM) has been used for in situ studies of laser- and

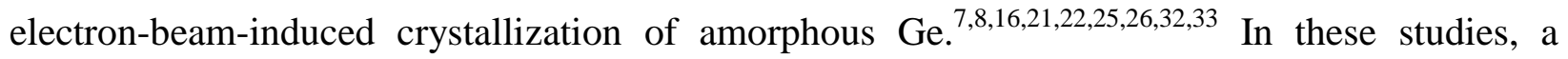
minute area of an electron transparent specimen of amorphous Ge is heated with a focused laser or electron beam, resulting in high local temperature gradients. A region of an electron transparent sample may be heated with a pulsed laser in nanoseconds and the crystallization of the heated region takes on the order of $1 \mu \mathrm{s}$, but it takes much longer for the temperature profile to homogenize, because the heat flow out is mainly through the plane of the electron transparent thin film (radiative cooling is insignificant on these timescales). ${ }^{34}$ Crystallization eventually grows unsteady and then quenches in the cooler parts of the film before the temperature gradient decays, thus the process is never in steady state - both the temperatures at the interfaces and the temperature gradient must change during the phase transformation.

Pulsed laser heating of an amorphous Ge TEM specimen results in a crystalline region with distinct microstructural features that may be divided into three zones, ${ }^{7,8,23,24}$ as seen in FIG. 1a. Zone I consists of nanocrystalline grains which nucleate and grow to impingement in tens of 
nanoseconds; ${ }^{23}$ formation of Zone I is complete within $\sim 100 \mathrm{~ns}$ of laser heating. ${ }^{8,22,23}$ The high nucleation rate in Zone $\mathrm{I}$ is assumed to occur because the Ge has exceeded the $\mathrm{T}_{\mathrm{ma}}$ during laser heating of the film while remaining significantly undercooled relative to the crystalline melting point. This is shown schematically in FIG. $1 \mathrm{~b}$ as the initial temperature profile, $\mathrm{t}_{1}$.

After the formation of Zone I, growth proceeds outward in the form of long, columnar grains with their major axes aligned in the direction of growth (Zone II). During in situ TEM experiments there are no current means to measure the instantaneous temperature profile within a laser-irradiated spot. A schematic of the assumed temperature profile in a cross section of the laser-irradiated thin film during liquid-mediated crystallization of Zone II is shown in FIG. $1 \mathrm{~b}$. The Zone II microstructure is similar to the columnar grain structure observed in steady-state experiments by Chojnacka ${ }^{4,5}$ and Grigoropoulos et al. ${ }^{6}$ where heat loss was controlled with the bulk substrate temperature which provided a large heat sink for the thin Ge film. The observed growth rates within Zone $\mathrm{II}^{7,8,25}$ are also similar to growth rates reported in references [4-6]. The existence of a transient liquid layer during explosive crystallization of Ge supported on heated bulk substrates has been confirmed by conductance measurements. ${ }^{5}$ To date, no similar independent confirmation of a liquid layer has been demonstrated for Zone II growth during in situ TEM experiments, but liquid-mediated growth is assumed due to both the high growth rates, which exclude the possibility of purely solid-state growth (based on extrapolation of experimentally determined solid state growth rates ${ }^{35}$ ), and due to the similarity of the resulting microstructure to experiments on heated substrates.

As crystallization proceeds toward cooler parts of the specimen, the columnar grain growth of Zone II transitions to a growth mode resulting in grains that spiral around the previously 
crystallized material (Zone III, FIG. 1a), the development of which during in situ TEM laser crystallization has been described in detail elsewhere. ${ }^{26}$

Zone II growth rates have been measured using photoemission TEM techniques. ${ }^{7,8,22,24-26,33}$ Dynamic TEM is one type of photoemission TEM technique in which a laser is used to induce a pulse of enough photoemitted electrons to form an image in just a few nanoseconds of exposure. A single electron pulse may be used to form an image and a series of electron pulses may be used to form a microsecond-scale multi-frame movie ("movie-mode" DTEM or MM-DTEM). The interframe time on the MM-DTEM instrument at Lawrence Livermore National Laboratory, where these experiments were performed, may be as little as $50 \mathrm{~ns}$ through the implementation of an electrostatic deflector installed below the electron optics that deflect the images to different parts of the camera detector. The deflection system allows the refresh rate of the camera to be overcome as the series of images is captured in a single camera exposure. The design, operation $^{36,37}$, and application ${ }^{38}$ of DTEM has been described elsewhere.

Both single frame DTEM and MM-DTEM have been used to study the crystallization kinetics of amorphous Ge. ${ }^{7,8,23-26}$ In a report on single frame DTEM experiments, ${ }^{24}$ Zone II growth in temperature gradient developed by a spatially Gaussian laser pulse, was reported to begin at $\sim 1 \mathrm{~m} / \mathrm{s}$, accelerate to more than $14 \mathrm{~m} / \mathrm{s}$, and then slow to $2 \mathrm{~m} / \mathrm{s}$ before transitioning to the Zone III growth mode. However, these experiments were limited to a single frame per crystallization experiment and direct measurement of front propagation rates was not possible. Later experiments using MM-DTEM and the same specimen heating $\operatorname{laser}^{8}$ with nine images for each experiment showed that Zone II growth rate did not change over time, even though the growth propagated through a high temperature gradient. Those MM-DTEM experiments showed a trend of decreasing growth rate with increasing local temperature gradient, but the data set was 
still fairly small. Here similar experiments are described where lower laser energies are used, just above the threshold that induces crystallization, filling out part of the experimental parameter space. At the crystallization threshold, Zone II can form without the formation of a distinct Zone I region. In this case, Zone II growth starts near the center of the regions heated by the Gaussian laser spot. Based on previous results, ${ }^{8}$ it was anticipated that this would result in Zone II growth beginning with a smaller negative temperature gradient than for higher laser pulse energies, which could lead to higher Zone II growth rates than previously observed in in situ TEM experiments.

\section{EXPERIMENTAL PROCEDURES}

In situ DTEM laser crystallization experiments were performed on 50-nm thick amorphous Ge films deposited at room temperature by magnetron sputtering onto commercially available 3 mm Si TEM supports with $0.25 \times 0.25 \mu \mathrm{m}$ windows spanned by 20 -nm thick silicon nitride membranes. A 99.999\% pure Ge target was used to deposit with $25 \mathrm{~W}$ dc power and Ar pressure of 2 mTorr. Crystallization was induced with a single laser pulse from a frequency doubled


Gaussian 12-ns full-width, half-maximum duration) directed onto the specimen $\sim 42^{\circ}$ from the Ge film normal. Pulse energies were varied from 3.0 to $3.6 \mu \mathrm{J}$. For laser pulses below $3 \mu \mathrm{J}$ single or even multiple laser pulses would not cause crystallization. The angle of incidence results in the crystallization of the Ge in an oval shape, as in FIG. 1a. The temperature profile established by the nanosecond-scale laser pulse does not decay significantly during the $\sim 2 \mu$ s duration of the in situ DTEM experiment, as shown in schematically in FIG. 1b and as a modelled with a finite element analysis (FEA) simulation in FIG. S1 (Supplemental Material).

Series of bright-field DTEM images of laser-induced crystallization events were generated 
from nine 20-ns electron pulses with an interframe spacing of $95 \mathrm{~ns}$. The delay between the specimen laser and the first electron image was varied from 100 to 500 ns. After crystallization was complete, conventional TEM images were taken of the crystallized region with the DTEM run in conventional thermionic mode.

In the nine frame MM-DTEM experiments where Zone I formed, the position of the Zone II crystallization front was determined at each time step on low magnification $(250 \times)$ images by fitting an ellipse to the crystalline Ge in ImageJ (https://imagej.nih.gov/ij/) and taking half of the minor axis of the ellipse as the position of the front relative to the center of the laser spot. In specimens where no Zone I formed, i.e. Zone II growth began from one or more isolated nucleation events. In these cases, the crystal growth was tracked from each grain only in the direction outward from the center of the laser-irradiated region and ignoring growth toward other crystallization grains. This makes the measurement as similar as possible to measurements on specimens with Zone I formation, where growth is only measured outward down the temperature gradient. When there are multiple grains, measuring each grain's growth in all directions would include growth toward the center of the laser spot - up the temperature gradient - and growth as the fronts of different grain approach each other, where local temperature of each would be affected by the released heat of crystallization of the other.

The sizes of Zone I (if present), II and III were measured on conventional TEM images taken after laser crystallization was complete by taking half of the minor axis of an ellipse fitted to the outer edge of each Zone. In addition to images taken after DTEM experiments, these measurements were also made on conventional TEM images of crystallized regions for which no time resolved movie was captured. 


\section{RESULTS}

Two examples of MM-DTEM imaging of Zone II crystallization induced by similar laser pulse energies are shown in FIG. 2, showing Zone II growth proceeding with (a) and without (b) Zone I formation. In FIG. 2a, Zone I has visibly formed by $100 \mathrm{~ns}$ at the center of the laserheated region, and in the remaining frames, Zone II growth proceeds. The formation of Zone III and quenching of crystal growth in cooler parts of the specimen occurs after the final frame. The complete crystallized region for this experiment is shown in FIG. 1a. In FIG. 2b, Zone I does not form, and by $100 \mathrm{~ns}$ there are only indistinct light spots in the right-hand side of the frame. By 195 ns, these spots may be unambiguously identified as crystalline regions and another crystalline grain has started to grow from a separate point several micrometers below the first grains. Growth proceeds from each of these grains in Zone II mode, even as new grains form (Fig. 2b, 385 ns frame).

A higher magnification image of a propagating crystallization front is shown in FIG. 3 . At the earliest time of Zone II growth shown (195 ns frame), the crystallization front has micronscale irregularities. These perturbations could be due to crystal-orientation-dependent variations in growth rate ${ }^{39}$ or local temperature variations. Some of the growth front perturbations appear to be unstable resulting in increasing differences in the local growth rate. For example, the motion of a straight section of the growth interface indicated with an arrow in the 385 ns frame of FIG. 3 is nearly arrested as the adjacent grains rapidly grow ahead of it and toward each other. A dark triangle is formed (indicated with an arrow in the $670 \mathrm{~ns}$ frame) where these grains have grown together, which later is a light triangle with a dark round form at the tip (arrow in the minutes after frame). This progression is interpreted as enthalpy of crystallization from the arrested grain and the two encroaching adjacent grains in a small area as adequate to cause a melt pool to form. 
The local temperature cannot be measured, but the formation of a dark rounded drop along with a bright adjacent region may be interpreted as the dewetting of the liquid portion of the Ge film from the silicon nitride before solidification. Bare silicon nitride is light and the rounded drop is dark due to mass-thickness contrast in the bright field image.

The region in the time-resolved images is indicated with a black circle in the lower magnification conventional TEM image. The liquid phase dewetting is well removed from the central (hottest) part of the laser-irradiated area, thus the melting must be due to the additional contribution of the heat of crystallization. Similar small dewet regions are found in several places in the Zone II region of the specimen shown here and also in other uncapped samples. It had not been observed in earlier experiments that used films capped with $7 \mathrm{~nm}$ of silica, ${ }^{8}$ because the solid silicon nitride support and the solid capping layer effectively suppress the thin film instability that causes dewetting to occur.

In all frames of Zone II growth in FIG 3., the edge of the crystalline regions appears to be bounded by a dark boundary, as other experiments performed at the same magnification and which is interpreted as the transient liquid phase which enables the rapid propagation of the growth front. The liquid phase is expected to appear darker than the amorphous phase in a bright field image (formed with an objective aperture), due to both increased thermal diffuse scattering and because liquid Ge has a higher density than the amorphous and crystalline solids. While it is expected that, if the image were slightly out of focus, Fresnel fringes may be seen at a crystalline-amorphous interface due to differences in density between the solid and amorphous phases (without a transient liquid layer), the fringe ahead to the front could be dark or light depending on whether the objective lens was underfocused or overfocused. In FIG 3. and similar 
experiments the boundary ahead of the crystal front always appears dark, supporting the interpretation of the image contrast as being due to the presence of a thin liquid layer.

The development of a dewet droplet during the crystallization process shown in FIG. 3 is additional strong visual indication that the subtly darker regions at the crystallization front are indeed evidence of a thin liquid phase. In previous in situ laser heating studies, the formation of such dark regions has been interpreted as evidence of the formation of a liquid. ${ }^{41,42}$ Here, the observation of droplet formation supports this interpretation of the image contrast. Highly localized formation of a liquid droplet also suggests that small perturbations of local growth front morphology may have a large impact on the local temperature profile.

The position of the Zone II growth front is plotted as a function of time for multiple MMDTEM experiments in FIG 4. For clarity, the nine separate crystallization experiments where Zone I formed are plotted in (a) and cases for the eight separate experiments where no Zone I formed are plotted in (b). In FIG. 4b, the growth front position for individual grains in a single crystallization experiment are plotted separately. All data sets were fit with straight lines. The slope represents the outward growth rate, which does not change significantly or systematically during the Zone II growth, even though both the temperature and temperature gradient at the growth front must change during the process given the Gaussian spatial profile of the heating laser pulse, as shown schematically in FIG. $1 \mathrm{~b}$.

In FIG. 5a, growth rates are plotted as a function of laser energy. The plot includes all the experiments from FIG. 4a-b and also includes previously published data ${ }^{8}$ for capped Ge films. The capped specimens were identical to those used in the experiments reported here except a 7 nm silica capping film had been deposited on top of the Ge. There is a large amount of scatter in the data with no clear trend relating growth rate and laser energy. 
As in previous experiments, ${ }^{8}$ it was observed that the same specimen laser energy may result in crystallized regions of different sizes and the sizes of the individual zones may vary considerably, suggesting variations in the energy absorbed during laser heating. This may be seen in FIG. S2 (Supplemental Material), which shows zone size as a function of laser energy measured on conventional TEM images. It may be seen that a single laser energy may result in different zone sizes, making the reliability of the laser energy as a proxy for relative temperature between experiments uncertain. In addition to using the laser energy as a measure of relative temperature, the size of Zone I may be also used. It is believed that Zone I forms where laser heating causes the film to exceed $T_{m a}$, thus the edge of Zone I should be close to $T_{m a}$, which allows the size of Zone I to serve as an indication of the amount of laser energy absorbed by the amorphous Ge film. Using the same approach as in reference [8], an attempt was made to consolidate the growth rate data by using the size of the crystalline region at $100 \mathrm{~ns}$ after the specimen was irradiated as a proxy for the relative temperatures of different samples. This is plotted in FIG. 5b, with data from the current experiments with uncapped Ge films and data from experiments using silica-capped Ge from [8]. Whereas, taken on its own, the data from [8] shows a slight trend of increasing growth rate with decreasing Zone I, the additional data makes it appear as if the growth rate is independent of both the laser energy and Zone I size, but with significant scatter.

The data point at the highest energy $(4.8 \mu \mathrm{J})$ is notably lower than the other data points, even given the amount of scatter. More data at the higher laser energies would have been desirable, but there is a practical upper limit to the laser energy that may be used in these thin film crystallization experiments. If the laser energy is above $\sim 4.5 \mu \mathrm{J}$, the center of the Ge film usually 
dewets extensively even when capped with silica. Extensive dewetting occurs at lower energies in the uncapped films.

\section{DISCUSSION}

\section{A. Growth rate trends}

Plotting growth rates against either the incident laser energy or Zone I size (FIG. 5) shows near constant behavior from the range of energies running from the threshold energy up to energies where extensive dewetting of the Ge thin film occurs. In previous work, ${ }^{8}$ the sparse data suggested a weak trend that Zone II growth rate decreased with increases in the size of Zone I. Given the Gaussian spatial profile of the beam, this was interpreted as potentially a change in the crystallization rate with changes in the local temperature gradient. The larger data set shows a growth rate of $\sim 11 \mathrm{~m} / \mathrm{s}$ with considerable scatter $( \pm 2 \mathrm{~m} / \mathrm{s})$ throughout the entire range of specimen laser energies used. The growth rate is similar to the plateaus observed by Grigoropoulos et al. ${ }^{6}(\sim 8-10 \mathrm{~m} / \mathrm{s})$ and Chojnacka and Thompson $(\sim 11-13 \mathrm{~m} / \mathrm{s}){ }^{4}$ Their data showed some scatter even though their experiments were designed to have steady state propagation of the explosive crystallization front for many microns and presumably, the temperature difference between the crystal-liquid interface and the liquid-amorphous interface is fixed. In the current experiments, steady state is never achieved. That the growth rates do not change significantly although the temperatures surrounding the crystallization front are changing suggests that when columnar explosive crystallization occurs, the growth rate plateaus. The high scatter in all these experiments may be due very highly localized temperature excursions caused by small perturbations in growth front morphology as was observed in FIG. 3.

Experimental investigations of explosive crystallization in silicon under non-steady state conditions have also shown plateauing of the liquid-mediated crystal growth at a maximal 
growth rate. ${ }^{18}$ Additionally, molecular dynamics simulations of liquid-mediated growth in both

Ge and Si by Albenze et al. ${ }^{15}$ led to the conclusion that explosive crystallization could robustly self-regulate and maintain a velocity that approaches a maximal growth rate through the variation in the thickness of the liquid layer. These experiments provide additional support for that conclusion.

\section{B. Model for Zone II growth}

Specimen and laser heating geometry is expected to impact the heat flow and thus the crystallization of amorphous material. With respect to the Zone II growth rates, these experimentally accessible range of laser energies are in a range where the growth rate apparently saturates and there is no systematic difference in the Zone II growth rate between capped and uncapped films. However, the presence of a capping layer still has an observable effect on the Zone II growth process. The Zone I size for the capped and uncapped films as a function of laser energy overlap in FIG. S2, suggesting the $7 \mathrm{~nm}$ silicon nitride capping layer has little impact on the absorption of the laser light. However, the differences in the capped and uncapped samples are apparent in the Zone II and Zone III sizes. The size of the entire crystallized region (Zone III size in FIG. S2) is distinctly larger for the uncapped than the capped, which can be attributed to the fact that capped films lose heat to the silica capping layer, quenching Zone III growth earlier than in uncapped films as discussed in [26]. The effect of the capping layer on Zone II is subtle in FIG. S2, but is clearly visible when the size of Zone II is plotted against the size of Zone I as in FIG. 6. Both capped and uncapped films show the same trend, but for the same Zone I size, the size of Zone II is consistently larger in the uncapped films. This is because the capping layer functions as heat sink, which limits how far Zone II growth can be sustained. While both samples 
release the same amount of latent heat during crystallization (same Ge thickness), the added heat capacity of the capping layer reduces the total rise in temperature.

To create a simple model for the expected extent of Zone II growth, the relationship between the amorphous melting point, $\mathrm{T}_{\mathrm{ma}}$, of $\mathrm{Ge}$ (assumed to be the threshold temperature required for Zone I formation), the size of Zone I, the size of Zone II, and the critical temperature to maintain Zone II propagation was developed. A very general expression for the assumed Gaussian temperature profile established in the electron transparent membrane by the incident laser as a function of radius from the center of the Gaussian laser spot incident on the sample, $r$, and the beam waist of the incident laser, $w\left(1 / \mathrm{e}^{2}\right.$ radius, $\sim 45 \mu \mathrm{m}$ here $)$, may be written:

$$
T=A \times \exp \left[-\frac{2 r^{2}}{w^{2}}\right]+T_{o}
$$

where $A$ is a constant that includes heat capacity (temperature-independent, for simplicity) and absorbed laser energy. $T_{o}$ is the temperature of the substrate before laser heating. If it is assumed that Zone I forms for any area that is raised above $T_{m a}$ during the initial laser heating, $A$ can be solved for in terms of $T_{m a}$ and the radius of Zone I, $R_{Z 1}$, which is taken to be at $T_{m a}$ :

$$
A=\left(T_{m a}-T_{o}\right) \times \exp \left[\frac{2 R_{Z 1}^{2}}{w^{2}}\right]
$$

In models of the heat transport for laser heating of the electron transparent thin films, it has been shown that thermal transport within the thin film does not significantly change the temperature profile in the specimen during the short period of time that the film is crystallizing, ${ }^{24,34}$ due to the high speed of the crystallization front, so it will be assumed that thermal transport within the film is insignificant for this problem. This is also consistent with our FEA discussed in the Supplemental Material (FIG. S1). If it is assumed that Zone II stops when the front drops below a critical temperature, $T_{c r i t}$, then one may develop an expression for the Zone II radius, $R_{Z 2}$, in terms of $T_{m a}, R_{Z 1}$, and $T_{c r i t}$ : 
$R_{Z 1}=\left[\frac{-w^{2}}{2} \times \ln \left(\frac{T_{c r i t}-T_{o}}{A}\right)\right]^{\frac{1}{2}}=\left\{\frac{-w^{2}}{2} \times \ln \left[\frac{T_{c r i t}-T_{o}}{T_{m a}-T_{o}} \exp \left(\frac{2 R_{Z 1}^{2}}{w^{2}}\right)\right]\right\}^{1 / 2}=\left[\frac{-w^{2}}{2} \times \ln \left(\frac{T_{c r i t}-T_{o}}{T_{m a}-T_{o}}+R_{Z 1}^{2}\right)\right]^{1 / 2}$

Everything is known except $T_{\text {crit }}$, so that may be fit to the data. This was performed for the measured Zone I and Zone II sizes from this experiment and from the data in reference [8]. The fitted functions are plotted with the data in FIG. 6. $\mathrm{T}_{\text {crit }}$ is found to be $800 \mathrm{~K}$ for the capped samples and $745 \mathrm{~K}$ for the uncapped specimens. If $\mathrm{T}_{\text {crit }}$ is the end of Zone II growth, that signals it is the transition to Zone III growth which is analogous to the transition between columnar and scalloped growth in the steady-state experiments by Chojnacka ${ }^{5}$ and Grigoropoulus et al. ${ }^{6}$ These fitted $\mathrm{T}_{\text {crit }}$ values are similar to the substrate temperatures where the transistion occurred betweeen scalloped and columnar growth, which were $\sim 700 \mathrm{~K}$ for $1.8 \mu \mathrm{m}$ films ${ }^{6}$ and about 690 $\mathrm{K}, 720 \mathrm{~K}$ and $750 \mathrm{~K}$ for $3.0 \mu \mathrm{m}, 1.8 \mu \mathrm{m}$, and $1.3 \mu \mathrm{m}$ films. ${ }^{5}$ The trend of thicker films exhibiting lower transition temperatures is also consistent with our observations. Thicker films have more material releasing heat during crystallization relative to the amount of material acting as a heat sink. Thus, they reach higher temperatures relative to thinner samples in the same way as discussed for our uncapped versus capped samples.

\section{SUMMARY AND CONCLUSIONS}

Laser crystallization of 50-nm amorphous Ge films was studied with MM-DTEM experiments with a focus on laser energies just above the crystallization threshold. Liquidmediated explosive crystal growth at relatively low laser energies were taken together with data from similar earlier experiments. Creating a larger dataset using multiple multi-frame DTEM experiments has led to a refinement of the observations of explosive crystallization in non-steady state conditions. The current work casts doubt on the previous hypothesis by Santala et al. that an increasing temperature gradient decreases the steady state liquid-mediated growth rate. Taking the current data with the previous data reported in [8] makes it seem far more likely that the 
DTEM measurements are in the range where there are bounded by the maximum crystal growth rate of Ge within the experimental error. The simple model for expected behavior of Zone II growth in the capped and uncapped Ge thin films is consistent with the behavior observed in these experiments and with critical temperatures observed in steady state experiments.

\section{SUPPLEMENTARY MATERIAL}

Details on the finite element analysis model of heat flow in Ge specimens during Zone II growth the DTEM experiments are available in the Supplementary Material. A plot of the sizes of Zones I, II, and III as a function of specimen laser pulse energy for both capped and uncapped Ge thin films is available in the Supplementary Material.

\section{ACKNOWLEDGMENTS}

Work performed by the LLNL participants was performed under the auspices of the U.S. Department of Energy, Office of Basic Energy Sciences, Division of Materials Sciences and Engineering for FWP SCW0974 by Lawrence Livermore National Laboratory under Contract DE-AC52-07NA27344. The authors thank the anonymous reviewer who provided detailed suggestions for the improvement of the manuscript. 


\section{FIGURES}

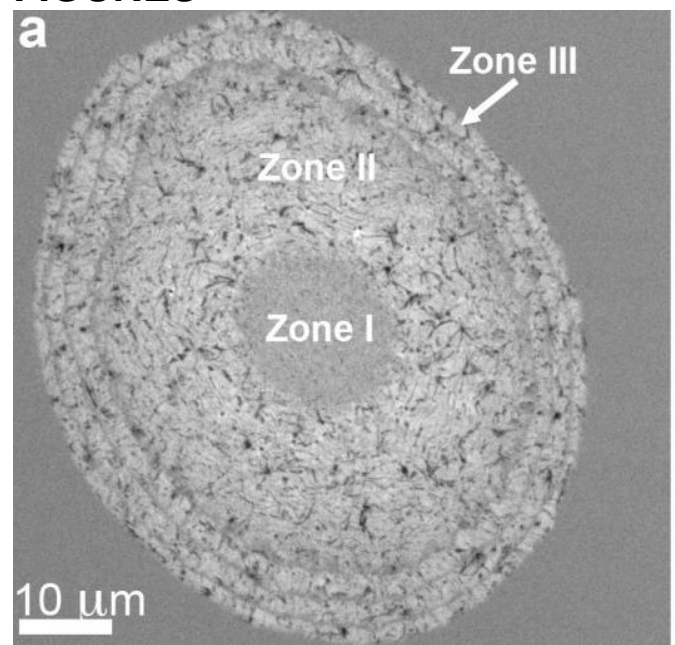

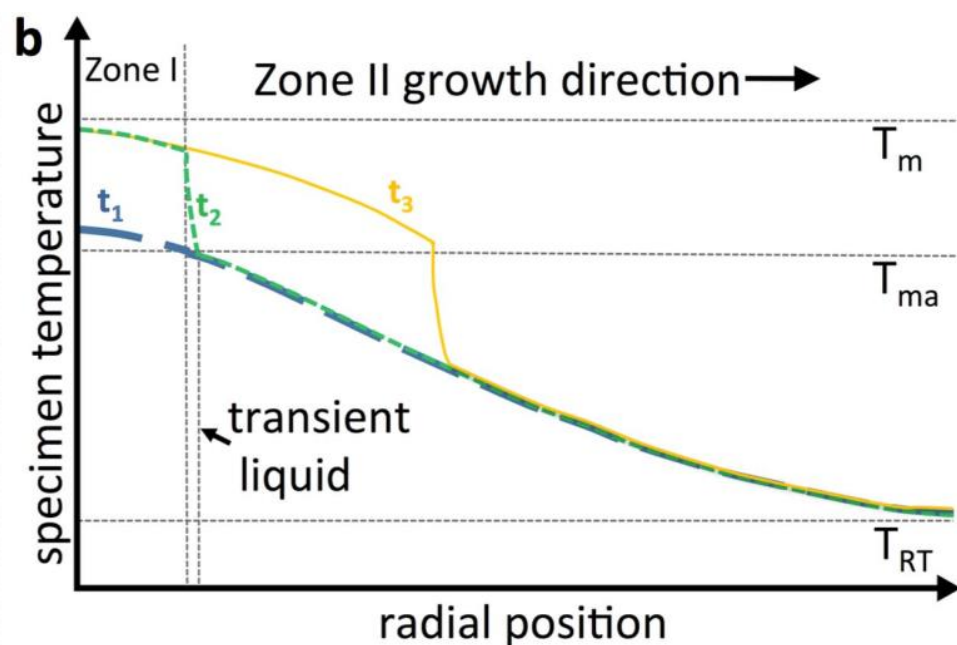

FIG. 1 (a) Crystalline zones resulting from exposing 50-nm amorphous Ge film supported on 20-nm amorphous silicon nitride film to a spatially Gaussian 12 -ns laser pulse incident $\sim 42^{\circ}$ from the film normal. (b) Schematic temperature profile based on a FEA model (Supplemental Material) at different stages of laser-initiated crystallization. The $\mathrm{t}_{1}$ line represents $\sim \mathrm{t}=0 \mathrm{~ns}$ from peak laser power when the film is heated but has not crystallized; $\mathrm{t}_{2}$ is immediately after Zone I formation showing a local increase in temperature due to the enthalpy of crystallization. Material adjacent to Zone $\mathrm{I}$ is raised above $\mathrm{T}_{\mathrm{ma}}$ and will crystallize, melting an adjacent layer of material; $t_{3}$ is $1-2 \mu$ s later near the end of Zone II growth.
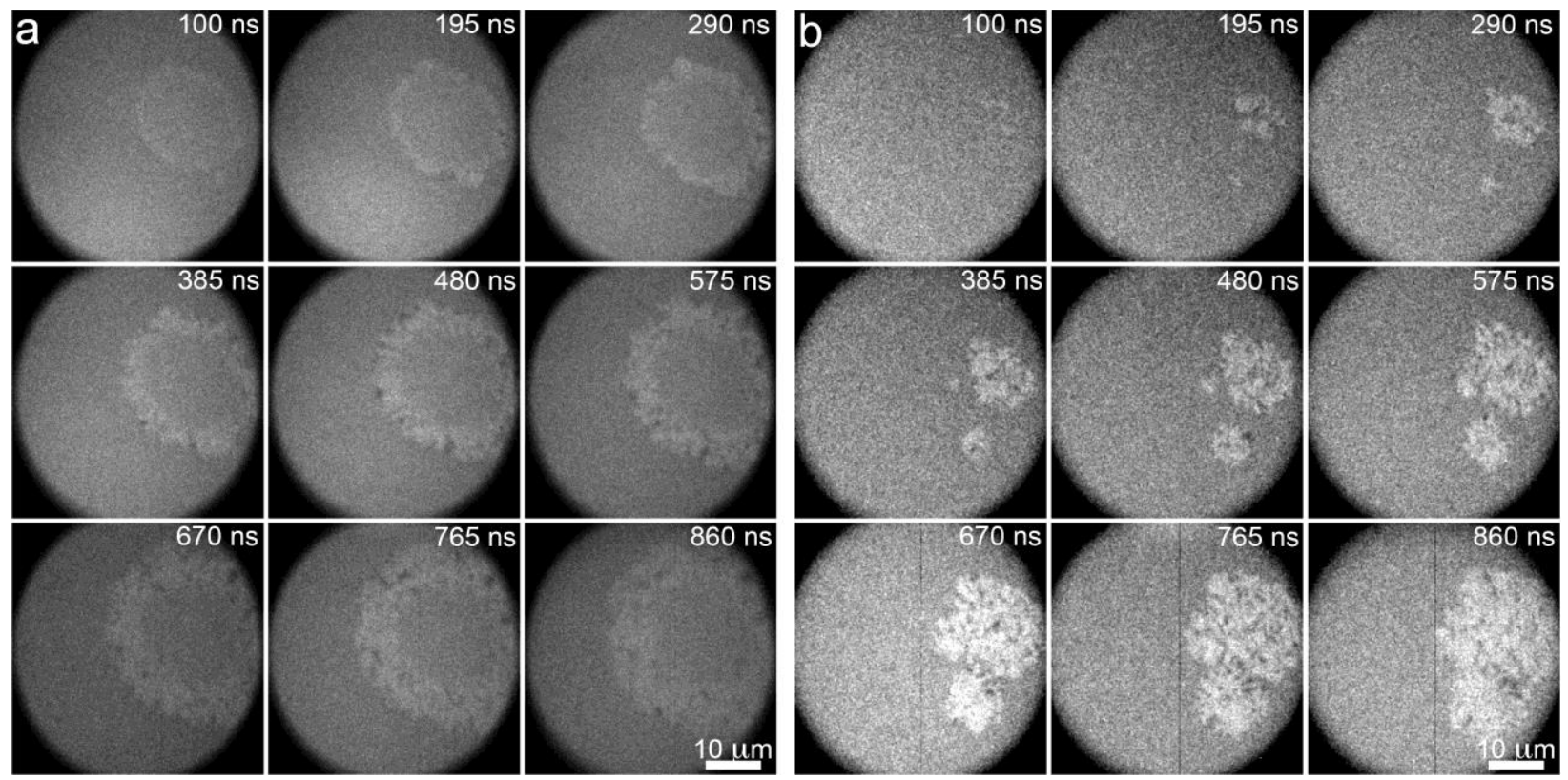

FIG. 2 Time-resolved images of laser-induced crystallization. The time signature in each frame indicates the time elapsed from the peak of the 12-ns laser pulse used to heat the specimen. (a) A crystallization event induced by a $3.40 \mu \mathrm{J}$ shot where Zone I forms before the initiation of Zone II growth (b) An example where a $3.44 \mu \mathrm{J}$ shot does not cause Zone I formation with its characteristic nanocrystalline grains. Instead, sparse nucleation events result in a few grains forming within $100 \mathrm{~ns}$ that grow to be several microns across. New grains nucleate and grow in later frames at separate sites. These examples are in a threshold range where Zone I formation may or may not occur. For laser pulses above $3.5 \mu \mathrm{J}$, Zone I always formed in these experiments (FIG 4. and FIG 5a), presumably because the absorbed energy was always adequate to raise the temperature above $\mathrm{T}_{\mathrm{ma}}$. 

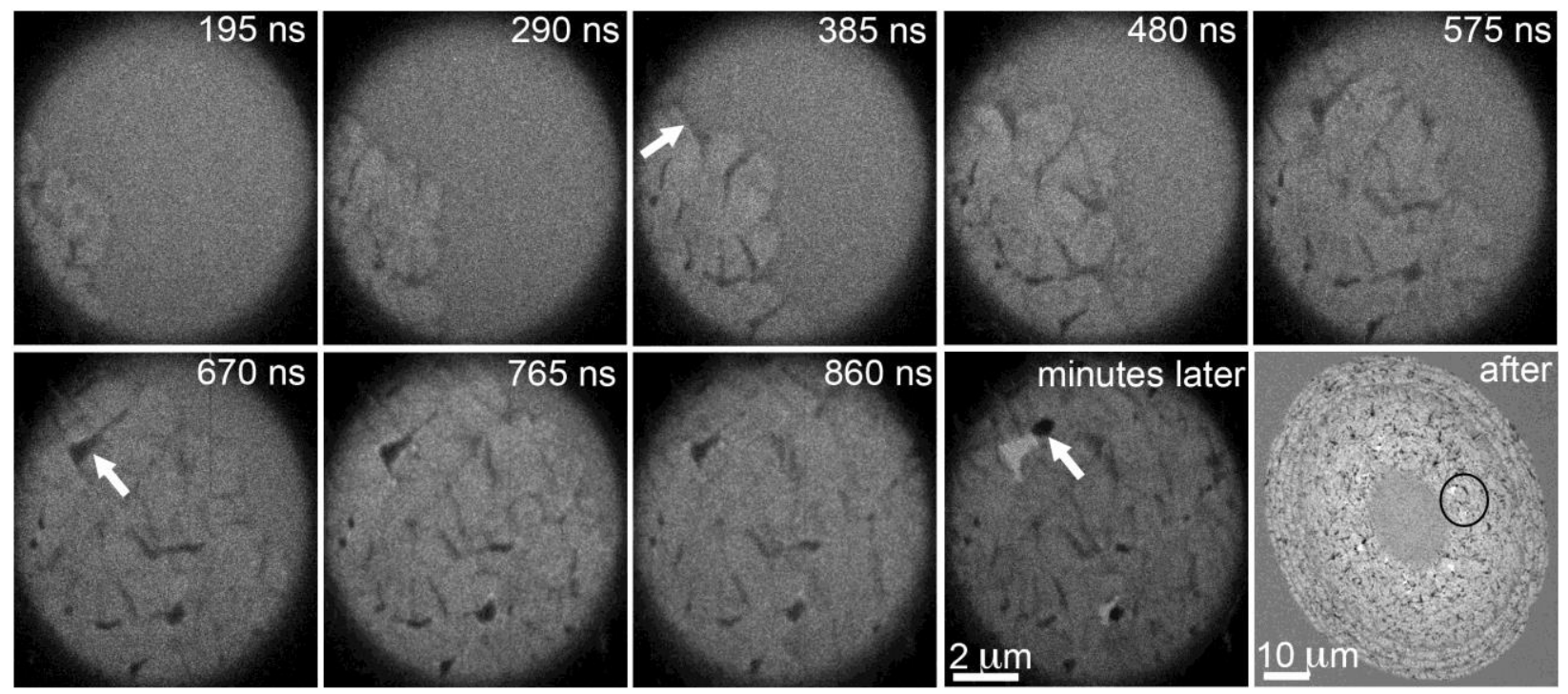

FIG. 3 Time-resolved images of Zone II growth during laser induced crystallization. The growth front has micronscale perturbations that may be due to local temperature variations or orientation-dependent differences in growth rates. As the perturbations grow they may further enhance the local temperature and growth rate variations. The motion of straight interface indicated with an arrow in the $385 \mathrm{~ns}$ frame is nearly arrested. Adjacent grains grow toward each other and the heat of crystallization causes a melt pool to form that dewets from the silicon nitride before solidification. The result of dewetting is indicated with arrows (minutes later frame): rounded Ge drops (dark) adjacent to areas bare silicon nitride (light); the mass-thickness contrast yields the highest contrast in the pulsed images. The region in the time-resolved images is indicated with a black circle in the lower magnification image.
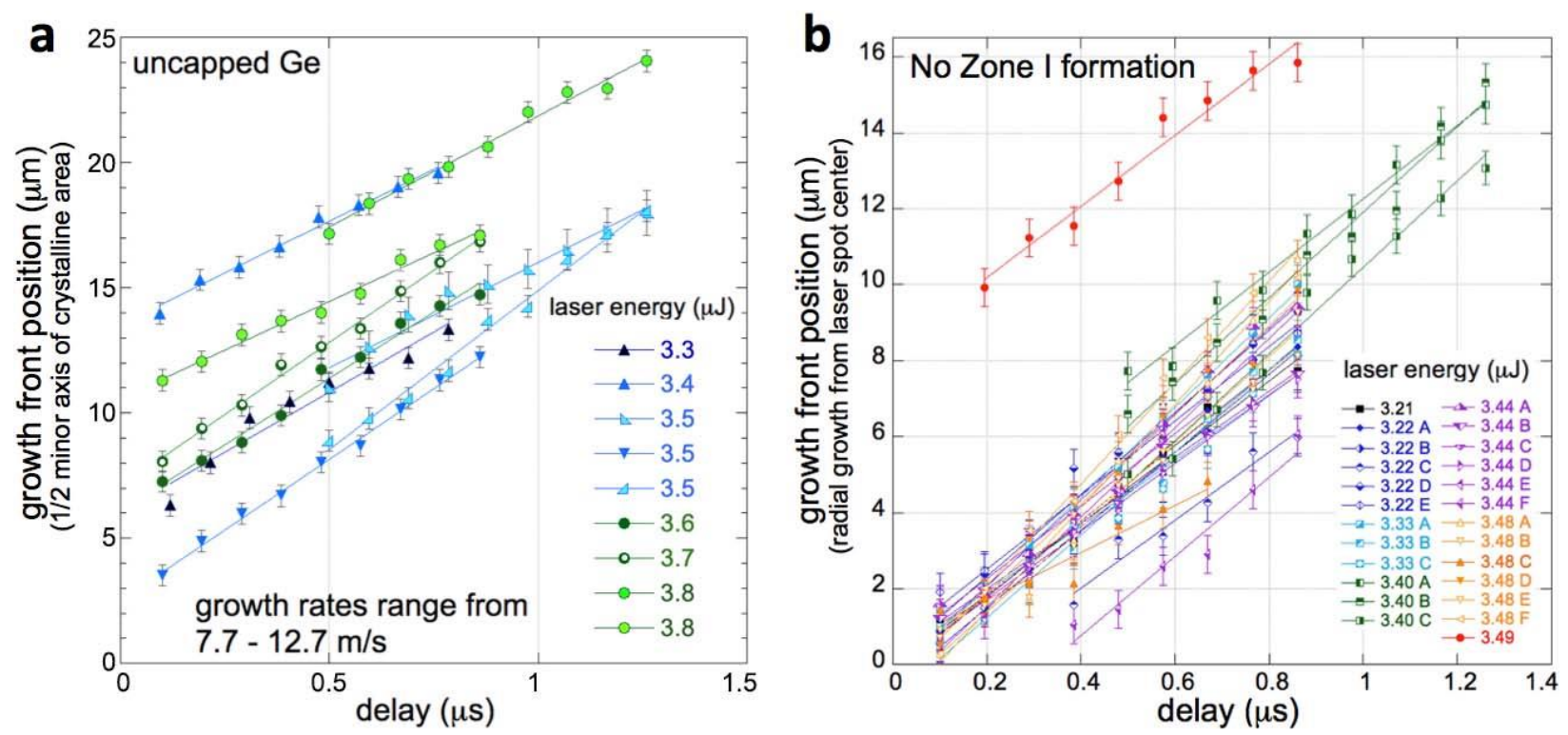

FIG. 4 (a) Growth front position $v s$ delay for crystal growth from Zone I for uncapped Ge thin films as in FIG. 2(a). (b) Growth front position $v s$ delay for crystal growth from grains without Zone I development. Multiple grains measured in a single experiment are designated with a single energy and a series of consecutive letters. The data for the specimen heated with a $3.49 \mu \mathrm{J}$ laser pulse does not project back to the origin, because multiple grains formed and impinged between the first two frames, growth was measured for the single coalesced region. 

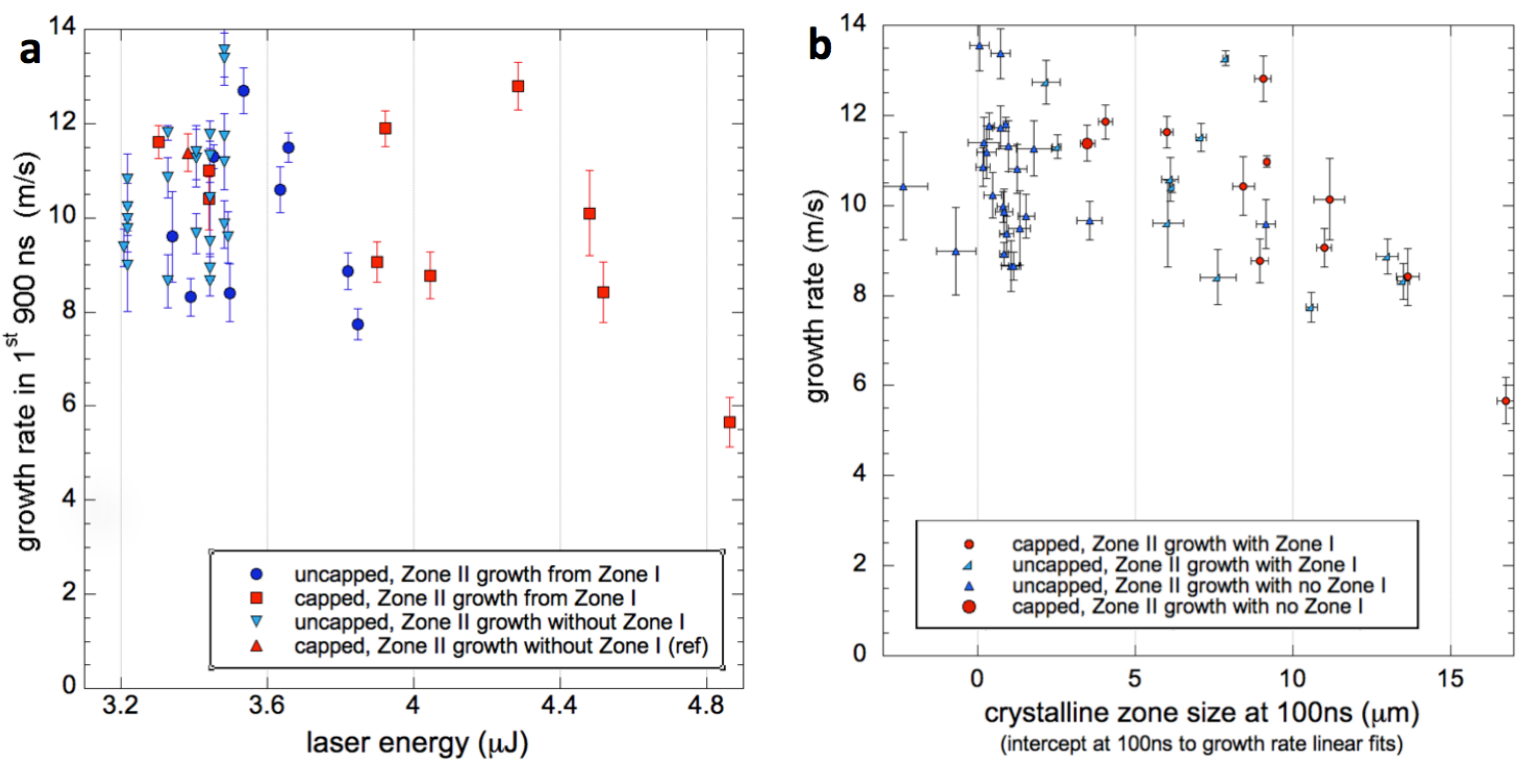

FIG. 5 (a) Growth rates during the first 900 ns of Zone II growth rate as a function of laser energy for all samples, both with and without Zone I. Different grains from the same sample in samples without zone one are given the same symbol and are aligned along the sample laser energy. (b) The growth rates in (a) are re-plotted against the Zone I size at $100 \mathrm{~ns}$. The data for the capped Ge films are from reference [8]. The two samples with negative values of crystalline Zone I size had no Zone I formation and the first grains became visible only well after $100 \mathrm{~ns}$, thus the $100 \mathrm{~ns}$ intercepts of the lines fit to their growth rates are negative.

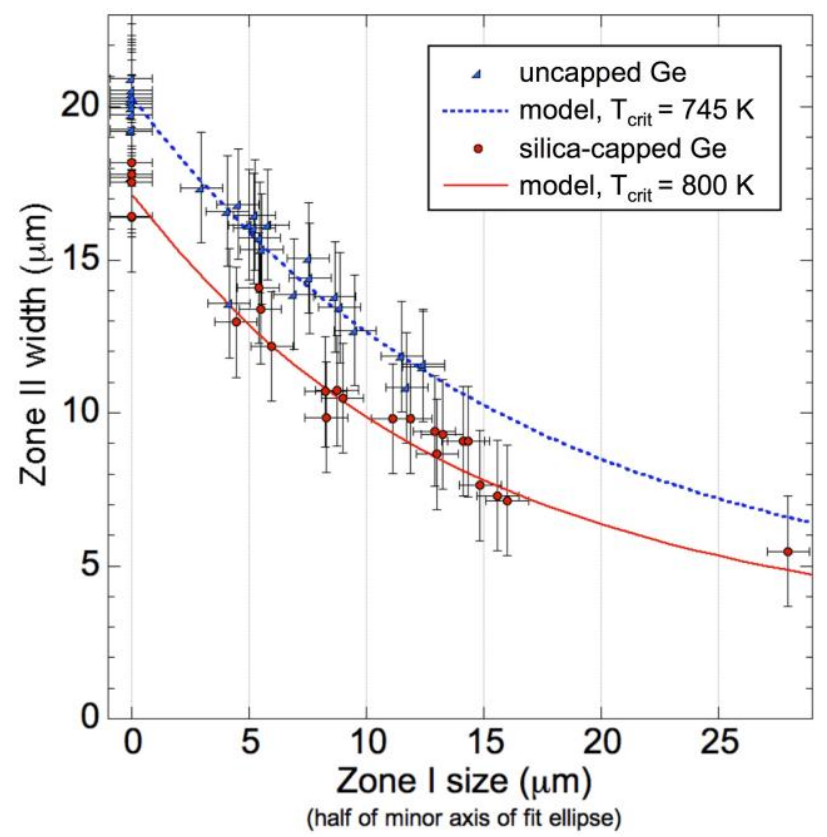

FIG. 6 Zone II width versus Zone I size for $50 \mathrm{~nm}$ thick capped and uncapped Ge. The data for silica-capped Ge are from reference [8]. 


\section{REFERENCES}

${ }^{1}$ J. Dore, D. Ong, S. Varlamov, R. Egan, and M. A. Green, IEEE Journal of Photovoltaics 4, 33 (2014).

2 J. L. Huang, S. Varlamov, J. Dore, J. S. Yun, and M. A. Green, Solar Energy Materials and Solar Cells 132, 282 (2015).

${ }^{3}$ N. H. Nickel, Laser Crystallization of Silicon, Vol. 75 (Elsevier, London, 2003).

${ }^{4}$ A. Chojnacka and M. O. Thompson, in Morphological instabilities during explosive crystallization of germanium films, 2001, p. P11.12.1.

${ }^{5}$ A. P. Chojnacka, Ph.D. dissertation Thesis, Cornell University, 2002.

${ }^{6}$ C. Grigoropoulos, M. Rogers, S. H. Ko, A. A. Golovin, and B. J. Matkowsky, Physical Review B 73 (2006).

${ }^{7}$ L. Nikolova, T. LaGrange, M. J. Stern, J. M. MacLeod, B. W. Reed, H. Ibrahim, G. H. Campbell, F. Rosei, and B. J. Siwick, Physical Review B 87, 064105 (2013).

${ }^{8}$ M. K. Santala, S. Raoux, and G. H. Campbell, Applied Physics Letters 107, 252106 (2015).

${ }^{9}$ J. C. C. Fan, H. J. Zeiger, R. P. Gale, and R. L. Chapman, Applied Physics Letters 36, 158 (1980).

10 B. G. Bagley and H. S. Chen, in A calculation of the thermodynamic first order amorphous semiconductor to metallic liquid transition temperature, Boston, 1978 (American Institute of Physics), p. 97.

11 F. Spaepen and D. Turnbull, in Kinetics of motion of crystal-melt interfaces, Boston, 1978 (American Institute of Physics), p. 73.

12 T. Takamori, R. Messier, and R. Roy, Applied Physics Letters, 201 (1972).

13 G. Gore, Philosophical Magazine Series 4 9, 73 (1855).

14 T. Takamori, R. Messier, and R. Roy, Journal of Materials Science 8, 1809 (1973).

15 E. J. Albenze, M. O. Thompson, and P. Clancy, Physical Review B 70, 094110 (2004).

16 O. Bostanjoglo, Physica Status Solidi a-Applied Research 70, 473 (1982).

17 M. O. Thompson, G. J. Galvin, J. W. Mayer, P. S. Peercy, J. M. Poate, D. C. Jacobson,

A. G. Cullis, and N. G. Chew, Physical Review Letters 52, 2360 (1984).

18 P. A. Stolk, A. Polman, and W. C. Sinke, Physical Review B 47, 5 (1993).

19 R. L. Chapman, J. C. C. Fan, H. J. Zeiger, and R. P. Gale, Applied Physics Letters 37, 292 (1980).

20 H. J. Leamy, W. L. Brown, G. K. Celler, G. Foti, G. H. Gilmer, and J. C. C. Fan, Applied Physics Letters 38, 137 (1981).

21 O. Bostanjoglo and G. Hoffmann, Physica Status Solidi a-Applied Research 73, 95 (1982).

22 O. Bostanjoglo, W. Marine, and P. Thomsenschmidt, Applied Surface Science 54, 302 (1992).

23 L. Nikolova, T. LaGrange, B. W. Reed, M. J. Stern, N. D. Browning, G. H. Campbell, J.

C. Kieffer, B. J. Siwick, and F. Rosei, Applied Physics Letters 97, 203102 (2010).

24 L. Nikolova, M. J. Stern, J. M. MacLeod, B. W. Reed, H. Ibrahim, G. H. Campbell, F. Rosei, T. LaGrange, and B. J. Siwick, Journal of Applied Physics 116, 093512 (2014).

25 T. T. Li, L. Bayu Aji, T. W. Heo, M. K. Santala, S. Kucheyev, and G. H. Campbell, Applied Physics Letters 108, 221906 (2016).

26 G. C. Egan, T. T. Li, J. D. Roehling, J. T. McKeown, and G. H. Campbell, Acta Materialia 143, 13 (2018). 

(1975).

F. M. Aymerich and A. Delunas, Physica Status Solidi a-Applied Research 31, 165

O. Bostanjoglo and G. Schlotzhauer, Physica Status Solidi a-Applied Research 68, 555 (1981).

30 M. M. Winseck, H.-Y. Cheng, G. H. Campbell, and M. K. Santala, Dalton Transactions 45, 9988 (2016).

31 R. Hultgren, D. Desai, T. Hwkins, and M. Gleiser, in Selected Values of the

Thermodynamic Properties of the Elements (Metals Park, Ohio, American Society for Metals, Metals Park, Ohio, 1973), p. 204.

32 R. K. Sharma, S. K. Bansal, R. Nath, R. M. Mehra, K. Bahadur, R. P. Mall, K. L. Chaudhary, and C. L. Garg, Journal of Applied Physics 55, 387 (1984).

33 O. Bostanjoglo and E. Endruschat, Physica Status Solidi a-Applied Research 91, 17 (1985).

34 M. K. Santala, B. W. Reed, S. Raoux, T. Topuria, T. LaGrange, and G. H. Campbell, Applied Physics Letters 102, 174105 (2013).

35 P. Germain, K. Zellama, S. Squelard, J. C. Bourgoin, and A. Gheorghiu, Journal of Applied Physics 50, 6986 (1979).

36 T. LaGrange, B. W. Reed, M. K. Santala, J. T. McKeown, A. Kulovits, J. M. K.

Wiezorek, L. Nikolova, F. Rosei, B. J. Siwick, and G. H. Campbell, Micron 43, 1108 (2012).

37 T. Lagrange, B. Reed, and D. J. Masiel, MRS Bulletin 40, 22 (2015).

38 G. H. Campbell, J. T. McKeown, and M. K. Santala, Applied Physics Reviews 1, 041101 (2014).

39 C. Reina, L. Sandoval, and J. Marian, Acta Materialia 77, 335 (2014).

40 R. Messier, T. Takamori, and R. Roy, Solid State Communications 16, 311 (1975).

41 J. S. Kim, T. LaGrange, B. W. Reed, R. Knepper, T. P. Weihs, N. D. Browning, and G. H. Campbell, Acta Materialia 59, 3571 (2011).

42 J. T. McKeown, K. Zweiacker, C. Liu, D. R. Coughlin, A. J. Clarke, J. K. Baldwin, J. W. Gibbs, J. D. Roehling, S. D. Imhoff, P. J. Gibbs, D. Tourret, J. M. K. Wiezorek, and G. H. Campbell, Journal of Materials 68, 985 (2016). 